\title{
Interval-valued contractive fuzzy negations
}

\author{
Benjamin Bedregal, Humberto Bustince, Glad Deschrijver, Radko Mesiar and Javier Fernandez
}

\begin{abstract}
In this work we consider the concept of contractive interval-valued fuzzy negation, as a negation such that it does increase the length or amplitude of an interval. We relate this to the concept of Lipschitz function. In particular, we prove that the only contractive interval-valued fuzzy negation is the one generated from the standard (Zadeh's) negation.
\end{abstract}

\section{INTRODUCTION}

Fuzzy sets theory, first introduced by Zadeh in [17], has shown itself a very valuable tool for problems that, by its very own nature, have to deal with imprecision, ignorance or vagueness. However, the definition of fuzzy set does not properly enough take into account this vagueness, since it imposes a single numerical value to measure the membership of an element to a given set. It can be very hard to provide precisely this numerical value, so it seems useful to find alternative ways of providing the information that do not require so much precision, or that, at least, allow to take into consideration somehow the imprecision or vagueness of the problem under consideration. In this sense, there have been made several proposals: among them we can find Atanassov's intuitionistic fuzzy sets theory ([2], [3]) and interval-valued fuzzy sets ([8]). In particular, the second cited extensions assigns to each element of the referential set not a number but a whole interval. The length of this interval can be understood ([7]) as a measure of the lack of knowledge or imprecision in the data that have to be handle in order to solve a given problem. For an application to image processing, see [5].

From this point of view, it is quite natural, form a theoretical as well as from a practical point of view, to consider those mappings such that the length of the resulting interval is at most as large as those of the input intervals. Or, from the lack of knowledge point of view, consider those processes such that the imprecision or lack of accuracy is not increased in the final result.

On the other hand, Lipschitzicianity is a very widely used concept in mathematical analysis. Basically, it imposes a restriction in the way a function can increase or decrease, and it occurs in fields as different as topology (fixed point maps) or the study of ordinary differential equations ([9]). So in some sense, it can also be understood as a mathematical

Benjamin Bedregal is with the Departamento de Informática e Matemática Aplicada, Universidade Federal do Rio Grande do Norte, Brazil, email: bedregal@dimap.ufrn.br.

Humberto Bustince and Javier Fernandez are with the Departament of Automatic and Computation, Public University of Navarra, email: \{bustince,fcojavier.fernandez\}@unavarra.es.

Glad Deschrijver is with the Department of Applied Mathematics and Computer Science, Ghent University, Belgium, email:Glad.Deschrijver@ugent.be

Radko Mesiar is with the Department of Mathematics and Descriptive Geometry, Slovak University of Technology, Bratislava, email:mesiar@math.sk way of preventing a function from increasing too much if the input variables remain close enough.

In this work we intend to bring together this two concepts. We will express the idea of not increasing the lack of ignorance by means of the Lipschitzicianity analytical tool. In particular this has led us to the concept of contractive interval-valued mapping. Clearly, this open a wide field of research, so in order to give a clear idea of what are we trying to do, we have focused in the specific case of interval-valued fuzzy negations. This particular instance, as simple as it can seem, provides in fact some valuable results. In particular, we prove that the only interval-valued fuzzy negation that does not increase the length of the input interval is also the only interval-valued fuzzy negation that preserves that length. And that negation is precisely standard negation. So, thanks to the connection by means of the concept of automorphism of Lipschitz functions and fuzzy negations, we prove that, basically, 1-Lipschitzicianity is equivalent to contractiveness for interval valued fuzzy negations.

Nevertheless, we want to stress that this work is only a first step in a very large field of possible research. For this reason, we have focused on the simple example of negations, that can be used as token for future developments of the theory, without involving too complicated issues.

The structure of this paper is the following. In the next section we present some preliminary definitions and results. In Section III we introduce interval-valued fuzzy sets and interval-valued mappings. In Section IV we present our main results on interval-valued contractive fuzzy mappings. In Section $\mathrm{V}$ we talk a bit of interval-valued $K$-Lipschitz mappings. We end with some conclusions.

\section{PRELIMINARIES}

In this section we recall the main concepts that we are going to use for our developments. We start by recalling what Lipschitz functions are.

\section{A. Lipschitz functions}

We recall here the mathematical concepts of Lipschitz function, as well as some properties that will be of interest for us.

Definition 1: A function $f:[0,1] \rightarrow[0,1]$ is called a Lipschitz mapping if there exists $K>0$ such that, for all $x, y \in[0,1]$, the inequality

$$
|f(x)-f(y)| \leq K|x-y|
$$

holds.

The smallest of such $K$ is called the Lipschitz constant of the mapping $f$. If $K$ is the Lipschitz constant of the mapping 
$f$, then $f$ is also called a $K$-Lipschitz mapping. In particular, 1-Lipschitz mappings are also called short maps.

It is worth to point out that the general definition of Lipschitz mapping is not restricted to the closed interval $[0,1]$, but considered over all real numbers. However, since we are going to deal with fuzzy negations, we take this domain-restricted definition.

With respect to the meaning of $K$-Lipschitzicianity, basically it does not allow $f$ to increase or decrease faster than a given rate with respect to the increase of the input variable. This rate is defined by the constant $K$. In this sense, it is clear that any $K$-Lipschitz mapping $f$ is also continuous. Moreover, it can be seen ([9]) that a $K$-Lipschitz mapping is differentiable almost everywhere with respect to the Lebesgue measure. In fact, $K$-Lipschitzicianity can also be understood as a sort of weak differenciability. Also observe that if a $K_{1}$-Lipschitz function and $g$ is a $K_{2}$ Lipschitz function, then $f \circ g(g \circ f)$ is a $K_{3}$-Lipschitz $\left(K_{4}\right.$ Lipschitz) function with $K_{3} \leq K_{1} K_{2}\left(K_{4} \leq K_{1} K_{2}\right)$.

For our following developments, we focus in bijective Lipschitz functions. In this sense, we start by introducing the concept of automorphism on the unit interval.

Definition 2: [6] A mapping $\varphi:[0,1] \rightarrow[0,1]$ is an automorphism if it is strictly increasing and bijective. We denote by $A u t([0,1])$ the set of all automorphisms over $[0,1]$.

Notice that for any automorphism $\varphi$ the identities $\varphi(0)=$ 0 and $\varphi(1)=1$ hold. Observe also that any automorphism is in particular continuous, but not necessarily $K$-Lipschitz, as the family of automorphisms $\varphi(x)=x^{p}$ with $p<1$ shows. On the other hand, we have the following result.

Proposition 1: Let $\varphi \in \operatorname{Aut}([0,1])$ be a $K$-Lipschitz automorphism. Then $K \geq 1$.

Proof. By definition

$$
|\varphi(1)-\varphi(0)|=1=1-0
$$

so the result is clear $\square$

Observe that the only important point for the proof is that $\varphi(1)=1$ and $\varphi(0)=0$, regardless which the other values of $\varphi$ are. In fact, not even monotonicity was necessary.

Example 1: Each of the automorphisms $\varphi_{p}(x)=x^{p}$ with $p \geq 1$ is $p$-Lipschitz. To see it, first of all notice that, from the mean value theorem, if $x>y$

$$
\varphi_{p}(x)-\varphi_{p}(y)=\varphi_{p}^{\prime}(c)(x-y) \leq p(x-y)
$$

for some $c \in(x, y)$. So $\varphi_{p}$ is Lipschitz with Lipschitz constant smaller than or equal to $p$. On the other hand,

$$
1-x^{p}-p(1-x) \rightarrow 0 \text { if } x \rightarrow 1,
$$

so the $p$-Lipschitzicianity follows.

Moreover, 1-Lipschitzicianity completely determines an automorphism, as the next result shows.

Proposition 2: An automorphism $\varphi \in \operatorname{Aut}([0,1])$ is 1Lipschitz if and only if $\varphi(x)=x$ for all $x \in[0,1]$.

Proof. From the 1-Lipschitzicianity of $\varphi$, we have, on one hand, that

$$
\varphi(x)=|\varphi(x)-\varphi(0)| \leq|x-0|=x
$$

whereas on the other hand

$$
1-\varphi(x)=|\varphi(1)-\varphi(x)| \leq|1-x|=1-x
$$

so we have that $\varphi(x) \geq x$, and the result follows from both inequalities

Remark. There is not a similar uniqueness result for $K$ Lipschitz automorphisms with $K>1$. To see it, fix $K>$ 1 , and $s \in] 0,1 / K[$. Then, the next automorphisms are $K$ Lipschitz:

$$
\phi_{s, 1}(x)=\min \left(K x, x \frac{1-K s}{1-s}+\frac{K s-s}{1-s}\right)
$$

and

$$
\phi_{s, 2}(x)=\max (K x-K+1,(1-K s) x) .
$$

Note that

$$
\sup \left\{\phi_{s, 1}(x) \mid s \in\right] 0,1 / K[\}=\min (K x, 1)
$$

is the upper bound of all $K$-Lipschitz automorphisms $\phi$ from Aut $([0,1])$. Nevertheless, it is not strictly monotone and thus not an automorphism.

On the other hand,

$$
\inf \left\{\phi_{s, 2}(x) \mid s \in\right] 0,1 / K[\}=\max (0, K x-K+1)
$$

is the lower bound of all $K$-Lipschitz automorphisms $\phi$ from $\operatorname{Aut}([0,1])$. As in the previous case, it is not strictly monotone and thus not an automorphism.

Of course, there is nothing specific from a mathematical point of view in the use of automorphisms. We can obtain a similar general result for any bijective continuous mapping, as the next result shows.

Proposition 3: Let $f:[a, b] \rightarrow[c, d]$ be a bijective $K$ Lipschitz mapping. Then $K \geq \frac{d-c}{b-a}$ and the only $\frac{d-c}{b-a}$ Lipschitz monotone bijection is

$$
f(x)=\frac{d-c}{b-a}(x-a)+c
$$

if $f$ is increasing, or

$$
f(x)=\frac{d-c}{b-a}(b-x)+c
$$

if $f$ is decreasing.

Proof. Suppose first that $f$ is increasing. Define the mapping $g:[0,1] \rightarrow[0,1]$ as

$$
g(x)=\frac{f((b-a) x+a)-c}{d-c}
$$

We have that

$$
\begin{array}{r}
|g(x)-g(y)| \leq\left|\frac{f((b-a) x+a)-c}{d-c}-\frac{f((b-a) y+a)-c}{d-c}\right| \\
=\left|\frac{f((b-a) x+a)}{d-c}-\frac{f((b-a) y+a)}{d-c}\right|
\end{array}
$$

and, since $f$ is $K$-Lipschitz, this is smaller than or equal to

$$
\frac{K}{d-c}|(b-a) x+a-(b-a) y-a|=K \frac{b-a}{d-c}|x-y|
$$


so $g$ is a Lipschitz automorphism. From Proposition 1, it follows that

$$
K \frac{b-a}{d-c} \geq 1
$$

or equivalently

$$
K \geq \frac{d-c}{b-a}
$$

as we intended to prove. If $K=\frac{d-c}{b-a}$, it follows from Proposition 2 that $g(x)=x$, and by clearing $f$ in the definition of $g$, the result follows.

Finally, if $f$ is decreasing, then the mapping $h(x)=$ $f(b+a-x)$ is increasing, and the results follows from the calculations for the increasing case

\section{B. Lipschitz fuzzy negations}

In this section we analyze the relation between the Lipschitz property and the concept of fuzzy negation. Further considerations on the subject, as well as related developments, can be found in [11].

We start recalling the concept of (fuzzy) negation.

Definition 3: A fuzzy negation is a nonincreasing mapping $N:[0,1] \rightarrow[0,1]$ such that $N(0)=1$ and $N(1)=0$.

If $N$ is continuous and strictly decreasing, i.e., if $x<y$ implies that $N(x)>N(y)$, then $N$ is called a strict negation. A strict negation $N$ which is involutive (i.e, such that $N(N(x))=x$ for all $x \in[0,1])$ is called a strong negation.

The most representative example of negation is the socalled standard (or Zadeh's) negation $N_{Z}(x)=1-x$. Moreover, if $\varphi \in \operatorname{Aut}([0,1])$ is any automorphism, then the mapping $N(x)=\varphi^{-1}(1-\varphi(x)$ defines a fuzzy negation. In fact, as proved by Trillas ([14]), for any strong negation $N$ there exists an automorphism $\varphi$ such that $N$ can can be written in the previous way.

As a first result, we show that there are not purely contractive fuzzy negations, i.e., $K$-Lipschitz fuzzy negations with $K<1$.

Proposition 4: Let $N$ be a $K$-Lipschitz fuzzy negation. Then $K \geq 1$.

Proof. The proof runs similarly to that of Proposition 1, just recalling the remark after that result $\square$.

Our aim is to see that the only 1-Lipschitz negation is Zadeh's negation. From Proposition 3 we have the following result.

Corollary 1: Let $N$ be a strict negation. Then $N$ is 1Lipschitz if and only if $N(x)=1-x$ for all $x \in[0,1]$. Proof. Just observe that, if $N$ is a strict negation, it is by definition a continuous, strictly decreasing bijection from $[0,1]$ to $[0,1]$. So, by Proposition 3 , it follows that

$$
N(x)=1-x
$$

for all $x \in[0,1]$

Now we want to drop out strictness. This can be done as follows.

Theorem 1: Let $N$ be a 1-Lipschitz negation. Then $N(x)=1-x$ for all $x \in[0,1]$.
Proof. Since $N$ is 1 -Lipschitz, we have that, for any $x \in[0,1]$

$$
1-N(x)=N(0)-N(x) \leq x
$$

so $N(x) \geq 1-x$. Analogously,

$$
N(x)-0=N(x)-N(1) \leq 1-x
$$

so $N(x) \leq 1-x$. The result follows

\section{INTERVAL-FUNCTIONS}

\section{A. Interval-valued fuzzy sets}

We start introducing some notations. Some more results can also be found in [8]. We denote by $L([0,1])$ the set of all closed subintervals of the closed unit interval $[0,1]$. That is:

$$
L([0,1])=\{\mathbf{x}=[\underline{x}, \bar{x}] \mid \underline{x}, \bar{x} \in[0,1] \text { and } \underline{x} \leq \bar{x}\}
$$

We denote by $0_{L}=[0,0]$ and $1_{L}=[1,1]$. We also define the mappings $l, r: L([0,1]) \rightarrow[0,1]$ given by

$$
l([\underline{x}, \bar{x}])=\underline{x}
$$

and

$$
r([\underline{x}, \bar{x}])=\bar{x}
$$

which provide the lower bound and the upper bound of the interval $\mathbf{x}=[\underline{x}, \bar{x}]$, respectively. Hence, given $\mathbf{x} \in L([0,1])$, we will denote $\underline{x}=l(x)$ and $\bar{x}=r(x)$.

We can consider in $L([0,1])$ two natural order relations. The first one is defined as $\mathbf{x} \leq_{L} \mathbf{y}$ if and only if $\underline{x} \leq \underline{y}$ and $\bar{x} \leq \bar{y}$. The second order relation is provided by the usual inclusion order, that is, $\mathbf{x} \subseteq_{L} \mathbf{y}$ if and only if $\mathbf{x} \subseteq \mathbf{y}$ as ordinary sets.

None of these orderings is complete. In order to solve somehow this problem, we are going to introduce the following order relation, considered by Yager and $\mathrm{Xu}$ ([]) among others. First of all, let $\mathbf{x} \in L([0,1])$. We define its score as $s(\mathbf{x})=\bar{x}+\underline{x}$. We also define its amplitude or length as

$$
W(\mathbf{x})=\bar{x}-\underline{x} .
$$

Then, given $\mathbf{x}, \mathbf{y} \in L([0,1])$ we will say that $\mathbf{x} \leq_{C} \mathbf{y}$ if and only if $s(\mathbf{x}) \leq s(\mathbf{y})$ or $s(\mathbf{x})=s(\mathbf{y})$ and $W(\mathbf{y}) \leq W(\mathbf{x})$. Notice that if $s(\mathbf{x})=s(\mathbf{y})$ and $W(\mathbf{y})=W(\mathbf{x})$, it follows that

$$
\underline{x}+\bar{x}=\underline{y}+\bar{y}
$$

and

$$
\bar{x}-\underline{x}=\bar{y}-\underline{y}
$$

so $\underline{x}=y$ and $\bar{x}=\bar{y}$. This means that any two intervals in $\bar{L}\left([0, \overline{1]})\right.$ are comparable by means of $\leq_{C}$. Moreover, we have the following immediate result.

Proposition 5: Let $\mathbf{x}, \mathbf{y} \in L([0,1])$. Then, if $\mathbf{x} \leq_{L} \mathbf{y}$ it also holds that $\mathbf{x} \leq_{C} \mathbf{y}$.

Proof. Just observe that if $\mathbf{x} \leq_{L} \mathbf{y}$, it follows that $s(\mathbf{x}) \leq$ $s(\mathbf{y})$

On the other hand, there is not such relation between $\leq_{C}$ and $\subseteq_{L}$. since, $[0,1]$ is the greatest element with respect to $\subseteq_{L}$, whereas $[0,1] \leq_{C}[1,1]$. 


\section{B. Interval mappings}

We start now recalling how the usual concept of continuity for real-valued mappings can be extended to the setting of interval mappings.

Let $f:[0,1] \rightarrow[0,1]$ be a mapping. Then, the mapping $\hat{f}: L([0,1]) \rightarrow L([0,1])$ given by

$$
\hat{f}(\mathbf{x})=[\inf \{f(x): x \in \mathbf{x}\}, \sup \{f(x): x \in \mathbf{x}\}]
$$

is well defined. Notice that $f$ is continuous if and only if $\hat{f}(\mathbf{x})=\{f(x): x \in \mathbf{x}\}([13])$.

In this paper we consider the following notions of continuity on $L([0,1])$.

(i) Moore continuity [12]. It is defined as an extension of the continuity on the set of the real numbers by considering the metric given by the distance between two intervals $\mathbf{x}, \mathbf{y} \in L([0,1])$, which is defined by: $d_{M}(\mathbf{x}, \mathbf{y})=\max \{|\underline{x}-\underline{y}|,|\bar{x}-\bar{y}|\}$.

(ii) Scott continuity. It is defined as an extension of the continuity in the set of real numbers, considering the quasi-metric $q_{S}(\mathbf{x}, \mathbf{y})=\max \{y-\underline{x}, \bar{x}-\bar{y}, 0\}$. It was introduced in [13] and [1]. An alternative way of defining the Scott continuity is to consider the $L([0,1])$ with the reverse inclusion order as a continuous domain [10]. Then, a function $f:(L([0,1]), \supseteq) \rightarrow(L([0,1]), \supseteq)$ is said to be Scott continuous if it is monotonic and preserves the least upper bound of directed sets. Remember that a directed set of $(L([0,1]), \supseteq)$ is a subset $S \subset(L([0,1]), \supseteq)$ such that every pair of intervals in $S$ has an upper bound in $S$.

The main result of [13] can be adapted to our setting as follows.

Theorem 2: Let $f:[0,1]^{n} \rightarrow[0,1]$ be a mapping. Then, the following items are equivalent.

(i) $f$ is continuous (with respect to the usual Euclidean metric);

(ii) $\hat{f}$ is Moore continuous;

(iii) $\hat{f}$ is Scott continuous.

\section{CONTRACTIVE INTERVAL-VALUED FUZZY NEGATIONS}

By analogy to fuzzy negations, interval-valued fuzzy negations can be defined as follows.

Definition 4: A mapping $\mathbf{N}: L([0,1]) \rightarrow L([0,1])$ is an interval-valued fuzzy negation if, for all $\mathbf{x}, \mathbf{y} \in L([0,1])$, the following properties hold.

(N1) $\mathbf{N}\left(0_{L}\right)=1_{L}$ and $\mathbf{N}\left(1_{L}\right)=0_{L}$;

$(\mathbf{N} 2 a)$ If $\mathbf{x} \leq_{L} \mathbf{y}$, then $\mathbf{N}(\mathbf{y}) \leq_{L} \mathbf{N}(\mathbf{x})$;

$(\mathbf{N} 2 b)$ If $\mathbf{x} \subseteq_{L} \mathbf{y}$, then $\mathbf{N}(\mathbf{x}) \subseteq_{L} \mathbf{N}(\mathbf{y})$;

If $\mathbf{N}$ also satisfies the involutive property

(N3) $\mathbf{N}(\mathbf{N}(\mathbf{x}))=\mathbf{x}$ for all $\mathbf{x} \in L([0,1])$

then $\mathbf{N}$ is said to be a strong interval-valued fuzzy negation.

A Moore (or Scott) continuous interval-valued fuzzy negation is said to be strict if it also satisfies the following properties.

$(\mathbf{N} 4 a)$ If $\mathbf{x}<_{L} \mathbf{y}$ then $\mathbf{N}(\mathbf{y})<_{L} \mathbf{N}(\mathbf{x})$
(N4b) If $\mathbf{x} \subset{ }_{L} \mathbf{y}$ then $\mathbf{N}(\mathbf{x}) \subset_{L} \mathbf{N}(\mathbf{y})$.

The following propositions in this section are proved in [4].

Proposition 6: A mapping $\mathbf{N}: L([0,1]) \rightarrow L([0,1])$ is an interval-valued (strict) fuzzy negation if and only if the mappings $\overline{\mathbf{N}}, \underline{\mathbf{N}}:[0,1] \rightarrow[0,1]$ defined as

$$
\overline{\mathbf{N}}(x)=r(\mathbf{N}([x, x])
$$

and

$$
\underline{\mathbf{N}}(x)=l(\mathbf{N}([x, x])
$$

are (strict) fuzzy negations and

$$
\mathbf{N}(\mathbf{x})=[\underline{\mathbf{N}}(\bar{x}), \overline{\mathbf{N}}(\underline{x})]
$$

for all $\mathbf{x} \in L([0,1])$.

Notice that $\underline{\mathbf{N}}(x) \leq \overline{\mathbf{N}}(x)$ for all $x \in[0,1]$.

Proposition 7: Let $N_{1}, N_{2}$ be (strict) fuzzy negations. If $N_{1}(x) \leq N_{2}(x)$ for all $x \in[0,1]$, then the mapping $I\left[N_{1}, N_{2}\right]: L([0,1]) \rightarrow L([0,1])$ defined by

$$
I\left[N_{1}, N_{2}\right](\mathbf{x})=\left[N_{1}(\bar{x}), N_{2}(\underline{x})\right]
$$

is an interval-valued (strict) fuzzy negation.

Notice that if $\mathbf{N}=I\left[N_{1}, N_{2}\right]$, then $\underline{\mathbf{N}}=N_{1}$ and $\overline{\mathbf{N}}=N_{2}$.

From now on, for any fuzzy negation $N$, we will denote $\hat{N}=I([N, N]$.

\section{A. Contractive fuzzy negations}

An usual critique to interval mathematics, which also is applied for interval fuzzy negations, is that the output interval can be very large and therefore not useful. In the following we formalize the class of interval fuzzy negations which has not this problem.

Definition 5: We say that an interval-valued fuzzy negation $\mathbf{N}$ is contractive if for all $\mathbf{x} \in L([0,1])$ the inequality

$$
W(\mathbf{N}(\mathbf{x})) \leq W(\mathbf{x})
$$

holds.

Example 2: The interval-valued fuzzy negation

$$
\hat{N}_{Z}(\mathbf{x})=[1-\bar{x}, 1-\underline{x}]
$$

is a contractive interval valued fuzzy negation. In particular, observe that

$$
W\left(\hat{N}_{Z}(\mathbf{x})\right)=W(\mathbf{x})
$$

for any interval $\mathbf{x} \in L([0,1])$. In fact, it is possible to prove ([5]) that this is the only interval valued fuzzy negation for which this preservation of the amplitude happens.

Our results on 1-Lipschitz negations allow us to characterize contractive interval-valued fuzzy negations. To start, we have the following result.

Theorem 3: Let $N$ be a strict fuzzy negation. Then $\hat{N}$ is a contractive interval-valued fuzzy negation if and only if $N(x)=1-x$ for all $x \in[0,1]$.

Proof. By definition, we have that

$$
\hat{N}(\mathbf{x})=[N(\bar{x}), N(\underline{x})] .
$$


So $\hat{N}$ is contractive if and only if

$$
N(\underline{x})-N(\bar{x}) \leq \bar{x}-\underline{x}
$$

for any $0 \leq \underline{x} \leq \bar{x} \leq 1$. But this is equivalent to say that $N$ is a 1-Lipschitz negation. So, by Theorem 1 , the result follows

Now we have the following result.

Theorem 4: Let $\mathbf{N}$ be an interval-valued fuzzy negation. Then, if $\mathbf{N}$ is contractive then $\mathbf{N}=\hat{N}$ for some strict fuzzy negation $N$

Proof. From Proposition 6, we know that

$$
\mathbf{N}(\mathbf{x})=[\underline{\mathbf{N}}(\bar{x}), \overline{\mathbf{N}}(\underline{x})]
$$

Since $\mathbf{N}$ is contractive, by taking $\mathbf{x}=[x, x]$ we arrive at

$$
\overline{\mathbf{N}}(x)=\underline{\mathbf{N}}(x)
$$

for all $x \in[0,1]$, so the result follows

Notice that the converse of this theorem does not hold. In fact, if we consider the strict negation $N(x)=1-x^{2}$, then $\hat{N}(\mathbf{x})=\left[1-\bar{x}^{2}, 1-\underline{x}^{2}\right]$. Since $\hat{N}([0.5,0.6])=[0.64,0.75]$, it follows that $\hat{N}$ is not contractive.

We can also prove the following result.

Theorem 5: Let $\mathbf{N}$ be an interval-valued fuzzy negation. Then $\mathbf{N}$ is contractive if and only if $\mathbf{N}=\hat{N}_{Z}$.

Proof. The proof is very similar to that of Theorem 3, using Proposition 6 and Theorem 4 this time

The following corollaries follows straightforward from the previous results.

Corollary 2: An interval-valued fuzzy negation $\mathbf{N}$ is contractive if and only if there exists a 1-Lipschitz strict fuzzy negation $N$ such that $\mathbf{N}=\hat{N}$.

Corollary 3: The unique strict (strong) contractive interval-valued fuzzy negation is

$$
\hat{N}_{Z}(\mathbf{x})=[1-\bar{x}, 1-\underline{x}] .
$$

\section{V. $K$-CONTRACTIVE INTERVAL-VALUED FUZZY NEGATIONS}

It is natural to consider a slightly more general definition of contractivity for interval-valued fuzzy negations, as follows.

Definition 6: Let $K \geq 1$. An interval-valued fuzzy negation $\mathbf{N}$ is called $K$-contractive if $W(\mathbf{N}(\mathbf{x})) \leq K W(\mathbf{x})$ for all $\mathbf{x} \in L([0,1])$.

Notice that, just by considering the negation of the interval $[0,1]$, it follows that the restriction $K \geq 1$ in the previous definition is not restrictive.

We have as a first result the following.

Proposition 8: Let $\mathbf{N}$ be a $K$-contractive interval-valued fuzzy negation. Then there exists a fuzzy negation $N$ such that

$$
\mathbf{N}=\hat{N} \text {. }
$$

Proof. Just notice that, if $\mathbf{N}([\underline{x}, \bar{x}])=\left[N_{1}(\bar{x}), N_{2}(\underline{x})\right]$, by taking $\bar{x}=\underline{x}=x$, from the $K$-contractivity, it follows that $N_{1}(x)=N_{2}(x)=x$ for all $x \in[0,1]$, as we wanted to prove $\square$
Now we can present the main result in this section.

Proposition 9: Let $\mathbf{N}=\hat{N}$ be an interval-valued fuzzy negation. Then $\mathbf{N}$ is $K$-contractive if and only if $N$ is $K$ Lipschitz.

Proof. Suppose first that $\mathbf{N}$ is $K$-contractive. From the $K$ contractivity, we have that

$$
W(\mathbf{N}([x, y]))=N(x)-N(y) \leq K(y-x),
$$

which is exactly the definition of $K$-Lipschitzicianity applied to the fuzzy negation $N$

On the other hand, if $N$ is $K$-Lipschitz, we have that

$$
W(\mathbf{N}([x, y]))=N(x)-N(y) \leq K(y-x)=K W([x, y])
$$

so the result follows $\square$

\section{CONCLUSIONS}

In this work we have studied and characterized the concepts of contractive and $K$-contractive interval-valued fuzzy negation. In particular, we have proved that the only intervalvalued fuzzy negation which is also contractive is precisely the interval-valued fuzzy negation created from Zadeh's negation.

Clearly, the developments in the current work are only a very first step in the way to extend the concept of Lipschitzicianity to the interval-valued setting. In this sense, the property of contractivity seems a good tool, although it would be probably necessary to refine further this definition. Nevertheless, we consider this approach quite promising, it seems Lipschitzicianity can be understood as a reinterpretation in terms of interval amplitudes, which is a key characteristic of intervals.

\section{REFERENCES}

[1] Acióly, B. M., and Bedregal, B. C. . A quasi-metric topology compatible with inclusion monotonicity on interval space. Reliable Computing, 3(3):305-313, 1997.

[2] Atanassov, K. T., Intuitionistic fuzzy sets. In: V. Sgurev, ed., VII ITKR's Session, Sofia, June 1983 (deposed in Central Science and Technical Library, Bulgarian Academy of Sciences, 1697/84, in Bulgarian).

[3] Atanassov, K. T. Intuitionistic Fuzzy Sets and Systems, 20, 87-96, 1986.

[4] Bedregal, B.C. . On interval fuzzy negations. Technical report, DIMAP/UFRN, 2009. (available at http://www.dimap.ufrn.br/ bedregal/main-publications.html).

[5] Bustince, H., Barrenechea, E., Pagola, M., and Fernandez, J. Intervalvalued fuzzy sets constructed from matrices: Application to edge detection. Fuzzy Sets and Systems, 160 (13), 1819-1840, 2009.

[6] Bustince, H., Burillo, P., Soria, F., Automorphisms, negations and implication operators. Fuzzy Sets and Systems, 134(2), 209-229, 2003.

[7] Bustince, H., Pagola, M., Barrenechea, E., Fernandez, J., Melo-Pinto, P., Couto, P., Tizhoosh, H.R., Montero, J. Ignorance functions. An application to the calculation of the threshold in prostate ultrasound images. Fuzzy Sets and Systems, 161(1), 20-36, 2010.

[8] Deschrijver. G., Kerre, E., On the relationship between some extensions of fuzzy set theory Fuzzy Sets and Systems, 133(2), 227-235, 2003.

[9] Evans, C, Gariepy, R.F., Measure theory and fine properties of functions, Studies in advanced mathematics, CRC Press, 1992.

[10] Gierz, G. , K. Hofmann, K. Keimel, J. D. Lawson, M. Mislove, and D. Scott. Continuous Lattices and Domains. Cambridge Press, Cambridge, 2003.

[11] Kolesarova, A., Mesiar, R., Lipschitzian De Morgan triplets of fuzzy connectives, submitted to Information Sciences.

[12] Moore, R. E. Methods and Applications of Interval Analysis. SIAM, Philadelphia, 1979. 
[13] Santiago, R. H. N., Bedregal, B. C. and Acióly, B. M. . Formal aspects of correctness and optimality in interval computations. Formal Aspects of Computing, 18(2):231243, 2006.

[14] E. Trillas, Sobre funciones de negación en la teoría de conjuntos difusos, Stochastica, III-1 (1979) 47-59 (in Spanish). English translation reprinted in: S. Barro A. Bugarin, A. Sobrino (Eds.), Advances in Fuzzy Logic, Universidad de Santiago de Compostela, 1998, pp. 31-43.

[15] R.R. Yager, On ordered weighted averaging aggregation operators in multicriteria decision-making, IEEE Transactions on Systems, Man and Cybernetics 18 (1988) 183-190.

[16] R.R. Yager, Families of OWA operators, Fuzzy Sets and Systems 59 (1993) 125-148.

[17] Zadeh, L. A., Fuzzy sets. Information Control, 8, 338-353, 1965. 